\title{
A priori bounds for periodic solutions of a class of Hamiltonian systems
}

\author{
VIERI BENCI AND PAUL H. RABINOWITZ
}

Istituto Matematica Applicata, Facolta di Ingegneria, Universita di Pisa, I-56100 Pisa, Italy; Department of Mathematics University of Wisconsin-Madison, Madison, WI 53706, USA

Abstract. This paper concerns estimates for periodic solutions of a very general class of Hamiltonian systems of prescribed energy. The estimtes are a priori upper and lower bounds for the action integral in terms of the period.

During the past few years, several papers have studied the existence and multiplicity of periodic solutions of a Hamiltonian system on a prescribed energy surface (see [1]-[10] and [12]-[17]). One of the difficulties in treating this question is that the period of such a solution is not known a priori. This note contains simple upper and lower a priori bounds for the period for a class of such problems which contains in particular most of the cases considered in [1]-[10] and [12]-[17]. (The remaining cases such as in [12] can be treated even more easily.)

To state these estimates more precisely, let $H \in C^{1}\left(R^{2 n}, R\right)$ and $p, q \in R^{n}$. The corresponding Hamiltonian system is

$$
\text { (i) } \dot{p}=-H_{q}(p, q), \quad \text { (ii) } \dot{q}=H_{p}(p, q) \text {. }
$$

To normalize matters, suppose we are interested in periodic solutions of (1) of energy 1 , i.e. the solutions lie on $\mathscr{D} \equiv H^{-1}(1)$. Assume there exists such a solution whose period is $T(>0)$. It is convenient to make the dependence of the equation on the period explicit. Therefore rescaling the time variable by $t \rightarrow 2 \pi T^{-1} t \equiv \lambda^{-1} t$, the period becomes $2 \pi$ and (1) transforms to

$$
\text { (i) } \dot{p}=-\lambda H_{q}(p, q), \quad \text { (ii) } \dot{q}=\lambda H_{p}(p, q) \text {. }
$$

Let $z=(p, q)$. The action integral associated with $(2)$ is

$$
A(z) \equiv \int_{0}^{2 \pi} p \cdot \dot{q} d t .
$$

Our main result is the following:

THEOREM. Suppose $H \in C^{1}\left(R^{2 n}, R\right)$ and satisfies $\left(H_{1}\right) \mathscr{D}$ is the boundary of a compact neighbourhood of 0 in $R^{2 n}$ and $H_{z}\left(\equiv\left(H_{p}, H_{q}\right)\right) \neq 0$ on $\mathscr{D},\left(\mathrm{H}_{2}\right) p \cdot H_{p}(z)>0$ for all $(p, q) \in R^{n}, p \neq 0$. If $z=(p, q)$ is a $2 \pi$ periodic solution of $(2)$ on $\mathscr{D}$, then there exist constants $\underline{a}, \bar{a}>0$ and independent of $z$ such that

$$
0<\underline{a} A(z) \leq \lambda \leq \bar{a} A(z) .
$$

Remarks. (i) Note that if $H(p, q)=K(p, q)+V(q)$, where the potential energy $V$ satisfies $\mathscr{R} \equiv\left\{q \in R^{n} \mid V(q) \leq 1\right\}$ is compact and $V_{q}(q) \neq 0$ on $\partial \mathscr{R}$, and the kinetic 
energy $K$ satisfies $K(0, q)=0, p \cdot K_{p}(p, q)>0$ if $(p, q) \in R^{n}$ and $p \neq 0$, and e.g. $K(\alpha p, q) \rightarrow \infty$ as $|\alpha| \rightarrow \infty$ uniformly for $q \in \mathscr{R}$ and $p \in S^{n-1}$, then $H$ satisfies $\left(\mathrm{H}_{1}\right)$ and $\left(\mathrm{H}_{2}\right)$. This special case contains the situations studied e.g. in [2], [7], [9], [13] and [15]-[17].

(ii) The upper and lower bounds for $\lambda$ involve the action integral. Experience has shown that such estimates are intimately related to corresponding existence results for (1). We conjecture that if $\left(\mathrm{H}_{1}\right)$ and $\left(\mathrm{H}_{2}\right)$ hold, there exists a periodic solution of (1) on $\mathscr{D}$.

Proof of the theorem. We will first prove (3) for the technically simpler case of $H \in C^{2}\left(R^{2 n}, R\right)$ and then show how the argument can be modified if $H \in C^{1}$. Note first that if $z(t)$ is a solution of $(2), p(t)$ cannot vanish identically on any open interval $\mathscr{I}$, for then by $\left(\mathrm{H}_{2}\right) H_{p}(0, q(t))=0$ for $t \in \mathscr{I}$ and $(0, q(t)) \in \mathscr{D}$. Consequently $H_{q}(0, q(t)) \neq 0$ for $t \in \mathscr{I}$ by $\left(\mathrm{H}_{1}\right)$ contrary to (2)(i). It follows that

$$
\int_{0}^{2 \pi} p(t) \cdot H_{p}(z(t)) d t>0 .
$$

Taking the scalar product of (2)(ii) with $p$ and integrating yields

$$
A(z)=\lambda \int_{0}^{2 \pi} p \cdot H_{p}(z) d t
$$

Hence $A(z)>0$ and

$$
A(z) \leq 2 \pi \lambda \max _{(\xi, \eta) \in \mathscr{D}} \xi \cdot H_{p}(\xi, \eta)
$$

The lower bound for $\lambda$ now follows from (5) and $\left(\mathrm{H}_{2}\right)$.

Next, taking the scalar product of (2)(i) with $H_{q}(z)$ shows

$$
\begin{aligned}
-\lambda \int_{0}^{2 \pi}\left|H_{q}\right|^{2} d t & =\int_{0}^{2 \pi} \dot{p} \cdot H_{q} d t \\
& =-\int_{0}^{2 \pi} p \cdot\left(H_{q p} \dot{p}+H_{q q} \dot{q}\right) d t \\
& =-\lambda \int_{0}^{2 \pi} p \cdot\left(H_{q q} H_{p}-H_{q p} H_{q}\right) d t
\end{aligned}
$$

or

$$
0=\lambda \int_{0}^{2 \pi}\left[\left|H_{q}\right|^{2}+p \cdot\left(H_{q p} H_{q}-H_{q q} H_{p}\right)\right] d t .
$$

Multiplying (6) by a parameter $b$ to be chosen later and adding to (4) gives

$$
A(z)=\lambda \int_{0}^{2 \pi}\left[p \cdot H_{p}+b\left|H_{q}\right|^{2}+b p \cdot\left(H_{q p} H_{q}-H_{q q} H_{p}\right) d t .\right.
$$

By $\left(\mathrm{H}_{1}\right)$ and $\left(\mathrm{H}_{2}\right)$ again, there is a constant $\gamma>0$ such that

$$
\left|H_{q}(0, \eta)\right| \geq \gamma \quad \text { if }(0, \eta) \in \mathscr{D} \text {. }
$$

Therefore, by the continuity of $H_{q}$, there is a constant $\sigma>0$ such that

$$
\left|H_{q}(\xi, \eta)\right| \geq \gamma / 2 \quad \text { if }(\xi, \eta) \in \mathscr{D} \text { and }|\xi| \leq \sigma .
$$


Decreasing $\sigma$ if necessary, it can also be assumed that

$$
\left|\xi \cdot\left(H_{q p}(\zeta) H_{q}(\zeta)-H_{q q}(\zeta) H_{p}(\zeta)\right)\right| \leq \gamma^{2} / 8
$$

if $\zeta=(\xi, \eta) \in \mathscr{D}$ and $|\xi| \leq \sigma$. Let $T_{1} \equiv\{t \in[0,2 \pi]|| p(t) \mid \leq \sigma\}, T_{2} \equiv[0,2 \pi] \backslash T_{1}$ and $l \equiv\left|T_{1}\right|$, where $|B|$ denotes the Lebesgue measure of the set $B$. By $\left(H_{2}\right),(8)$ and (9),

$$
\begin{aligned}
I_{1} & \equiv \int_{T_{1}}\left[p \cdot H_{p}+b\left|H_{q}\right|^{2}+b p \cdot\left(H_{q p} H_{q}-H_{q q} H_{p}\right)\right] d t \equiv \int_{T_{1}} \mathscr{H} d t \\
& \geq b\left(\frac{\gamma^{2}}{4}-\frac{\gamma^{2}}{8}\right) l=\frac{b \gamma^{2} l}{8} .
\end{aligned}
$$

Letting

$$
M_{1}=\max _{\zeta=(\xi, \eta) \in \mathscr{D}}\left|\xi \cdot\left(H_{q p}(\zeta) H_{q}(\zeta)-H_{q q}(\zeta) H_{p}(\zeta)\right)\right|
$$

and

$$
\omega \equiv\left(2 M_{1}\right)^{-1} \min _{\zeta \in \mathscr{D},|\xi| \geq \sigma} \xi \cdot H_{p}(\zeta)
$$

it follows that

$$
I_{2} \equiv \int_{T_{2}} \mathscr{H} d t \geq(2 \pi-l) M_{1}(2 \omega-b) .
$$

Choosing $b=\omega$ yields

$$
I_{2} \geq(2 \pi-l) M_{1} \omega
$$

Since

$$
\lambda^{-1} A(z)=I_{1}+I_{2},
$$

(10) and (12) imply

$$
\lambda^{-1} A(z) \geq \omega\left(\frac{\gamma^{2}}{8} l+(2 \pi-l) M_{1}\right) \geq 2 \pi \omega \min \left(\frac{\gamma^{2}}{8}, M_{1}\right)
$$

and the upper bound for $\lambda$ in (3) follows from (14). Thus (3) is proved for $H \in C^{2}$.

An examination of the above argument shows $\underline{a}$ depends on $C^{1}$ bounds for $H$ on $\mathscr{D}$ while $\bar{a}$ depends on $M_{1}$ and therefore on $C^{2}$ bounds for $H$ on $\mathscr{D}$. Consequently a better upper bound for $\lambda$ is needed to establish the $C^{1}$ version of (3). Let $W(z)$ be a $C^{1}$ function in a neighbourhood of $\mathscr{D}$ with values in $R^{n}$. Taking the scalar product of (1)(i) with $W$ and arguing as in (6) yields

$$
0=\lambda \int_{0}^{2 \pi}\left[H_{q}(z) \cdot W(z)+p \cdot\left(W_{p}(z) H_{q}(z)-W_{q}(z) H_{p}(z)\right)\right] d t .
$$

Suppose $W$ satisfies

$$
W(\zeta) \cdot H_{q}(\zeta) \geq \gamma^{2}
$$

if $\zeta=(0, \eta) \in \mathscr{D}$. Then choosing $\sigma$ so that

$$
W(\zeta) \cdot H_{p}(\zeta) \geq \gamma^{2} / 4
$$

if $\zeta=(\xi, \eta) \in \mathscr{D}$ and $|\xi| \leq \sigma$ and arguing as in (9)-(14) gives

$$
\lambda^{-1} A(z) \geq 2 \pi \bar{\omega} \min \left(\gamma^{2} / 8, \bar{M}_{1}\right),
$$


where

$$
\bar{M}_{1} \equiv \max _{\zeta=(\xi, \eta) \in \mathscr{D}}\left|\xi \cdot\left(W_{p}(\zeta) H_{q}(\zeta)-W_{q}(\zeta) H_{p}(\zeta)\right)\right|
$$

and

$$
\bar{\omega}=\left(2 \bar{M}_{1}\right)^{-1} \min _{\zeta \in \mathscr{D},|\xi| \geq \sigma} \xi \cdot H_{p}(\zeta) .
$$

Thus (3) is established for this case provided that there exists a $C^{1}$ function $W$ satisfying (16).

A simple way to obtain $W$ is to use a notion due to Palais [11]. If $E$ is a real Banach space, $O \subset E$ is open and $\Phi \in C^{1}(O, R)$, then $w \in E$ is said to be a pseudogradient vector for $\Phi$ at $e \in \mathcal{O}$ if

$$
\text { (i) }\|w\|_{E} \leq 2\left\|\Phi^{\prime}(e)\right\|_{E^{*}}, \quad \text { (ii) }\left\langle\Phi^{\prime}(e), w\right\rangle_{E^{*}, E} \geq\left\|\Phi^{\prime}(e)\right\|_{E^{*}}^{2},
$$

where $\langle\cdot, \cdot\rangle$ denotes the pairing between $E^{*}$ and $E$. If $\Phi \in C^{1}(E, R)$, $\tilde{E}=\left\{e \in E \mid \Phi^{\prime}(e)=0\right\}, W(e)$ is a pseudogradient vector for $\Phi$ for all $e \in \tilde{E}$ and $W$ is locally Lipschitz continuous on $\tilde{E}$, then $W(\cdot)$ is called a pseudogradient vector field on $\tilde{E}$. Palais proved [11]:

LeMMA. If $\Phi \in C^{1}(E, R)$, there exists a pseudogradient vector field $W$ for $\Phi$ on $\tilde{E}$.

Choosing $E=R^{n}$ and $\Phi=H(0, q)$, this lemma implies there is a pseudogradient vector field $W(q)$ for $H(0, q)$ on $\tilde{E}$. By $\left(H_{1}\right),\left|H_{q}(\zeta)\right| \geq \gamma>0$ for $\zeta=(0, \eta) \in \mathscr{D}$. Hence $\tilde{E} \cap\left\{q \in R^{n} \mid(0, q) \in \mathscr{D}\right\}=\varnothing$ and by (18)(ii)

$$
H_{q}(0, \eta) \cdot W(\eta) \geq\left|H_{q}(0, \eta)\right|^{2} \geq \gamma^{2}
$$

for $(0, \eta) \in \mathscr{D}$ so (16) holds. Finally the proof of the lemma shows that if one uses a smooth partition of unity in the construction given there, $W$ is smooth and in particular can be assumed to be $C^{1}$.

Acknowledgements. This research was sponsored in part by the National Science Foundation under grant \# MCS-8110556 and the United States Army under contract \# DAAG29-80-C-0041. Reproduction in whole or in part is permitted for any purpose of the US Government.

\section{REFERENCES}

[1] A. Ambrosetti \& G. Mancini. On a theorem by Ekeland and Lasry concerning the number of periodic Hamiltonian trajectories. J. Differential Equations 43 (1981), 1-6.

[2] V. Benci. Closed geodesics for the Jacobi metric and periodic solutions of prescribed energy of natural Hamiltonian systems. Ann. Inst. H. Poincaré, Anal. nonlin.

[3] H. Berestycki, J. M. Lasry, G. Mancini and B. Ruf. Existence of multiple periodic orbits on star-shaped Hamiltonian surfaces. Comm. Pure Appl. Math. 38 (1985), 253-290.

[4] F. Clarke. A classical variational principle for periodic Hamiltonian trajectories. Proc. Am. Math. Soc. 76 (1979), 186-188.

[5] 1. Ekeland \& J. M. Lasry. On the number of periodic trajectories for a Hamiltonian flow on a convex energy surface. Ann. Math. 112 (1980), 283-319.

[6] M. Girardi. Multiple orbits for Hamiltonian systems on starshaped surfaces with symmetries. Ann. Inst. H. Poincaré, Anal. nonlin. 1 (1984), 285-294.

[7] H. Gluck \& W. Ziller. Existence of periodic solutions of conservative systems. Seminar on Minimal Submanifolds. Princeton University Press (1983), 65-98. 
[8] E. W. C. van Groesen. Existence of multiple normal mode trajectories on convex energy surfaces of even classical Hamiltonian systems. J. Differential Equations 57 (1985), 70-89.

[9] K. Hayashi. Periodic solution of classical Hamiltonian systems. Tokyo J. Math. 6 (1983), 473-486.

[10] H. Hofer. A simple proof for a result of I. Ekeland and J. M. Lasry concerning the number of periodic Hamiltonian trajectories on a prescribed energy surface. Preprint.

[11] R. S. Palais. Critical point theory and the minimax principle. Proc. Symp. Pure Math. 15. AMS, Providence, RI (1970), 185-212.

[12] P. H. Rabinowitz. Periodic solutions of Hamiltonian systems. Comm. Pure Appl. Math. 31 (1978), 157-184.

[13] P. H. Rabinowitz. Periodic solutions of a Hamiltonian system on a prescribed energy surface. J. Differential Equations 33 (1979), 336-352.

[14] P. H. Rabinowitz. On the existence of periodic solutions for a class of symmetric Hamiltonian systems. J. Nonlin. Anal. - TMA.

[15] O. R. Ruiz. Existence of brake orbits in Finsler mechanical systems. Springer Lecture Notes in Mathematics 597 (1977), 542-567.

[16] H. Seifert. Periodische Bewegungen mechanischen Systeme. Math. Z. 51 (1948), 197-216.

[17] A. Weinstein. Periodic orbits for convex Hamiltonian systems. Ann. Math. 108 (1978), 507-518. 